\title{
Construction of Light-trees for WDM Multicasting under Splitting Capability Constraints
}

\author{
Zsigri, Anikó; Guitton, A.; Molnár, M.
}

Published in:

Proceedings of 10th International Conference on Telecommunications

Link to article, DOI:

10.1109/ICTEL.2003.1191206

Publication date:

2003

Document Version

Publisher's PDF, also known as Version of record

Link back to DTU Orbit

Citation $(A P A)$ :

Zsigri, A., Guitton, A., \& Molnár, M. (2003). Construction of Light-trees for WDM Multicasting under Splitting Capability Constraints. In Proceedings of 10th International Conference on Telecommunications (Vol. 1). IEEE. https://doi.org/10.1109//CTEL.2003.1191206

\section{General rights}

Copyright and moral rights for the publications made accessible in the public portal are retained by the authors and/or other copyright owners and it is a condition of accessing publications that users recognise and abide by the legal requirements associated with these rights.

- Users may download and print one copy of any publication from the public portal for the purpose of private study or research.

- You may not further distribute the material or use it for any profit-making activity or commercial gain

- You may freely distribute the URL identifying the publication in the public portal 


\title{
Construction of Light-trees for WDM Multicasting under Splitting Capability Constraints
}

\author{
Anikó ZsIGRI \\ Research Center COM, Technical University of Denmark \\ DK-2800 KGS.Lyngby, Denmark \\ az@com.dtu.dk \\ Alexandre GurTTON \\ IRISA/University of Rennes I, Campus de Beaulieu \\ 35042 RENNES Cedex, France \\ alexandre.guitton@irisa. fr \\ Miklós MOLNÁR \\ IRISA/INSA, Campus de Beaulieu \\ 35042 RENNES Cedex, France \\ miklos.molnareirisa.fr
}

\begin{abstract}
Communication systems with all-optical multicasting have better performance than those using opticalvelectricaloptical conversion. Multicast protocols assume that all nodes in the network can forward the signal from one input to several outputs. Since to fabricate an optical switch with splitting capability is an expensive technology, there are few switches that are multicast capable. The heuristics designed for all-optical networks have to handle this limitation. In this paper, we introduce a Shortest Path Based Forest algorithm for all-optical networks. We propose a post-processing algorithm to reduce the number of wavelengths needed. The effect of this post-processing algorithm is also examined in a well-known approach
\end{abstract}

Keywords: All-optical networks; Multicast; WDM; Splitting capability; Routing algorithms.

\section{INTRODUCTION}

The technology of optical networks is continuously changing, leading to better performance. The optical transmission links used to be simple one wavelength point-to-point connections with optical transmitters and receivers. Today this type of optical links are being replaced with WDM (Wavelength Division Multiplexing) transmission links to increase the capacity. WDM is also known as Optical Frequency Division Multiplexing, since multiple wavelengths are transmitted simultaneously and combined on the same optical fiber. WDM technology is very attractive for high-capacity networks, such as backbone networks, since many data streams can be transmitted on the same fiber in parallel. Transmission systems with 10.2 Tbps on a fiber have been demonstrated [1]. This capacity was obtained using 273 wavelengths, and each wavelength had $40 \mathrm{Gbps}$ transmission rate. Presently many WDM transmission systems are available from different providers.

Multicast communication is becoming increasingly important in the recent years, due to more efficient bandwidth usage and the increasing popularity of the point-multipoint multimedia applications. In the network layer, multicast support means that a multicast protocol is running on the router, so packets are generally forwarded on tree. Tunneling can be used to handle multicast incapable routers [2].

While the duty of the network layer is to deliver multicast routes (create the multicast tree), the responsability of the physical layer is to realize the transmission on the optical network. In order to support multicasting in the optical domain, the optical switch has to be able to transmit one incoming signal to several outputs, which requires the usage of power-splitters. Having splitters in the switch induces insertion loss and power division loss. This power loss has to be compensated with amplifiers. Because of the fabrication complexity of the desired components, the multicast capable switches are very expensive and therefore rarely implemented into existing systems.

In many cases the splitting problem is avoided with optical/electrical/optical conversion (O/E/O) [3], but this solution is not so efficient, since the electrical domain limits the data rate and introduce delay. Another solution would be to use tapping devices in the switches with

0-7803-7661-7/03/\$17.00@2003 IEEE 
special multicast tree generation algorithms [4]. This solution avoids the need of power-splitters, but requires more wavelengths. In this paper we will focus on alloptical solutions and rely on the currently implemented switch architectures.

IP layer multicasting with physical layer unicasting avoids the use of $\mathrm{O} / \mathrm{E} / \mathrm{O}$ converters and the splitting ability of the switches. Measurements show that all-optical multicasting can reduce the number of wavelengths with $60 \%$ compared to optical unicasting [5]. All-optical trees are realized by light-trees, which are point-to-multipoint optical connections [6]. This concept reduces the number of transceivers and the average packet hop distance in the network. The set of light-trees generated is called light-forest.

In this paper we assume that when a node can split, it has infinite splitting capability. A node with splitting capability is called multicast capable (MC) node, while a node without splitting capability is called multicast incapable (MI) node in the literature [7]. A network, in which all switches are MC nodes is a network with full splitting capability, while a network with MI nodes is a sparse splitting capable network. The full splitting capable network with wavelength conversion capability can handle the same multicast trees as the electronical domain. In case of sparse splitting capability or lack of wavelength converters, the multicast tree has to be realized differently in the optical domain than in the electronical domain. In this paper sparse splitting capable networks with full wavelength conversion capability are discussed.

Finding a tree with minimal total cost in sparse splitting capable networks is referred to as a Degree Constrained Steiner Problem (DCSP) [8]. This theoretical graph problem is a generalization of the Steiner Problem, and is therefore also NP-complete [9]. While an optimal tree for the Steiner Problem always exists, this is not the case for the DCSP problem, that is why forests have to be used.

\section{MATHEMATICAL BACKGROUND}

Consider a connected network $G=(V, E)$, where $V$ is a set of vertices and $E$ a set of edges, a cost function with positive value $d(e)$ on edges $e \in E$, a source, $s$, and a group, $Z$, of destinations. In order to realize multicasting, the construction of a tree spanning $Z \cup\{s\}$ is needed. Generally, two kind of multicast trees are used:

- shortest path trees,

- minimum Steiner trees.

Shortest path trees are designed to minimize the delay from the source $s$ to each destination, or member, $m \in$

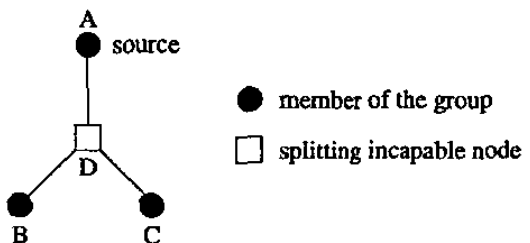

Fig. 1. Unsolvable topology for Minimum Steiner Tree Problem and for Shortest Path Based Tree Problern

$Z$. Let the shortest path from $s$ to $m$ be path $(s, m)$. The Shortest Path Based Tree can then be defined as:

$$
T=\bigcup_{m \in Z} \operatorname{path}(s, m) \text {. }
$$

This problem can be solved by using the Dijkstra algorithm [10].

The total cost $d(T)$ of a tree, $T$, is defined as the sum of the cost of all edges in $T$ :

$$
d(T)=\sum_{e \in T} d(e)
$$

The Minimum Steiner tree is the tree $T^{*}$ with minimal cost $d\left(T^{*}\right)$ spanning $Z \cup\{s\}$. Finding $T^{*}$ is NP-complete [11], that is why heuristics are frequently used.

In sparse splitting capable optical networks, finding a Shortest Path Based Tree or a Minimum Steiner tree is not always solvable because of $M I$ nodes. In Figure 1, a tree spanning $A, B$ and $C$ can not be found due to the splitting incapability of the $D$ branching node. A forest has to be built in order to obtain a solution. In the following, the Degree Constrained Shortest Path Based Forest Problem and the Degree Constrained Steiner Problem are described.

\section{A. Degree Constrained Shortest Path Based Forest Prob- lem}

Finding a forest of Shortest Path Based Trees, spanning $Z \cup\{s\}$ and respecting node constraints, will be called the Degree Constrained Shortest Path Based Forest Problem (DCSPF). This problem is an extension of the Shortest Path Tree Problem. The DCSPF can always be solved by using the set of shortest paths between the source and the destination. The goal is to find a forest that:

- minimizes the number of Shortest Path Based Trees (DCSPF-1),

- minimizes the total cost (DCSPF-2).

By reduction to the Set Covering Problem, it can be shown that DCSPF-1 is NP-complete. By reduction to the Weighted Set Covering Problem, it can be shown that DCSPF-2 is also NP-complete, so heuristics should be 
used. Heuristics we will propose will be for the DCSPF1 problem.

\section{B. Degree Constrained Steiner Problem}

This problem is first referred in [8]. Given the set $M I S$ of MI nodes, a minimum total cost forest with branching nodes not in MIS has to be found. Many heuristics exist for this problem, such as the modification of Takahashi-Matsuyama algorithm [12], called Member-Only (MO) [5]. Some heuristics for these two problems are mentioned in the following section.

\section{HEURISTICS FOR SPARSE SPLITTING CAPABLE NETWORKS}

Three metrics have been considered in order to compare different heuristics:

- number of wavelengths,

- number of wavelength channels,

- average delay from the source to the members.

Since the number of wavelengths is limited on each link, it is important to minimize the number of wavelengths needed by a forest. On a forest, some edges can belong to several trees. These edges must have different wavelengths. The number of wavelengths is defined as the maximum number of trees sharing the same edge. The number of wavelength channels in a forest is equal to the sum of the total number of edges on each tree.

There are two important categories of algorithms used for IP multicast routing:

- Heuristic approaching Minimum Steiner trees,

- Shortest Path Based Tree algorithms.

Most of the existing heuristics in all-optical networks approach Minimum Steiner trees. A Minimum Steiner tree minimizes the total cost of the partial spanning tree.

MO is a well-known heuristic for sparse splitting capable networks [5]. It is a modification of the TakahashiMatsuyama heuristic. First, the source is connected to the closest member by a shortest path, creating a tree. In each iteration, the closest node to the tree is connected either to a splitting capable node or to a leaf node, by a shortest path.

\section{A. Shortest Path Based Forest heuristic}

We propose an algorithm, which is in the Shortest Path Based Tree algorithm category. It is called Shortest Path Based Forest algorithm (SP). The original version of this algorithm is used by MOSPF and is not able to handle the splitting capability of switches [2]. We have modified the Shortest Path Based Tree algorithm to take into account the splitting capability of nodes. We propose two main steps for SP to provide a good solution. The

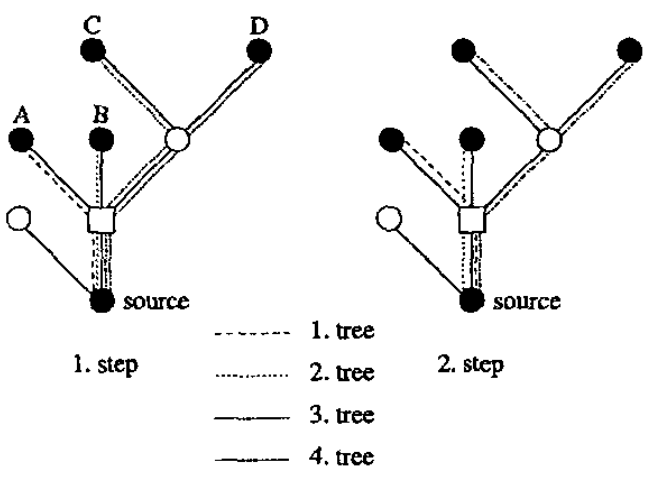

Fig. 2. Spanning trec created by the Shortest Path Based Forest heuristic.

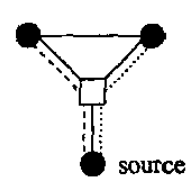

(a) SP heuristic

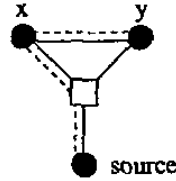

(b) S3P heuristic
Fig. 3. Comparisons of studied heuristics - (a): SP (b): S3P.

first is the construction of the shortest pathes from the source to the destinations. In step two, pathes are merged together if the resulting tree only contains MC branching nodes. The created forest covers the same edges as the shortest pathes. On Figure 2, three trees are needed to handle the splitting incapability constraints. On the first step, 4 trees are created, since there are 4 destinations. Only the path from the source to $\mathrm{C}$ and the path from the source to D can be merged on the second step.

The SP algorithm is optimal for the delay metric and solutions can be found in polynomial time. It can generate more trees. The trees can be overlapped on several edges, which increase the number of needed wavelengths. This shortcoming can be improved with a post-processing algorithm. In the next section we propose a post-processing algorithm, which improves the feature of SP.

\section{B. Post Processing Algorithm}

The post-processing algorithm (PP) is designed to reduce the number of wavelengths needed by the forest built by our proposed heuristic, SP. The PP algorithm applied on the SP algorithm will be called S3P.

Certain trees are merged together using shortest path in order to decrease the number of wavelengths (since the number of trees decreases). Two trees are merged 
only if two leaves $x$ and $y$ satisfy:

$$
d(x, y) \leq \min \{d(x, \text { source }), d(y, \text { source })\},
$$

where $d(x, y)$ is the distance between $x$ and $y$ in the tree.

Each merging induces a loop, which has to be removed. PP removes the longest possible path to keep the tree structure. This path has the following properties:

- The extremities have to be either members or having degree greater or equal than 3 in the tree.

- All internediate members are non-members and having degree 2 .

algorithm PP
begin
forall member $x$ do
find the closest member $y$ to $x$ such as
$(x, y \in M C S$ or $x, y$ are leaves $)$ and $T_{x} \neq T_{y}$
if $d(x, y) \leq \min \{d(x$, source $), d(y$, source $)\}$
then
if $T_{x}$ and $T_{y}$ can be connected by the
shortest path from $x$ to $y$ then
$T_{x y} \leftarrow T_{x} \cup T_{y} \cup$ path $(x, y)$
remove from $T_{x y}$ the loop by removing
the longest path
delete $T_{x}$ from the forest
delete $T_{y}$ from the forest
add $T_{x y}$ to the forest
endif
endif
endforall
end

In most of the cases, each merge reduces the number of wavelength channels or the number of wavelengths used by the forest. Figure 3 shows the behavior of the S3P algorithm. $d(x, y)$ is lower than $\min \{d(x$, source $), d(y$, source $)\}$, so the tree $T_{y}$ containing $y$ connects to $T_{x}$ through $x$. The number of wavelengths used and the number of wavelength channels are reduced by one.

This algorithm does not assure the optimal reorganization of the forest. Its advantages manifests in the simplicity and easy implementation. A more efficient PP algorithm can be obtained as follows. Instead of merging trees in $G$, a new graph $G^{\prime}$ is created. In $G^{\prime}$, each tree of the forest is represented by a node. A shortest path between a member $x$ of a tree $T_{x}$ and a member $y$ of a tree $T_{y}$ is represented in $G^{\prime}$ by an edge $e$ from the node corresponding to $T_{x}$ to the node corresponding to $T_{y}$. The weight of $e$ is set as:

$$
d(e)==d(p a t h(x, y))-d(l(x, y)),
$$

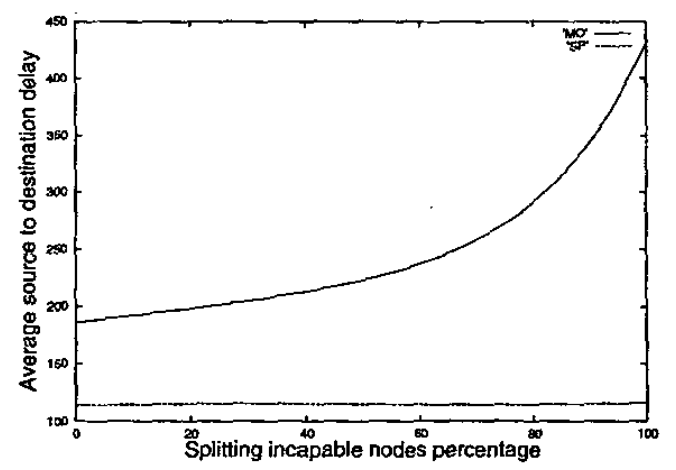

Fig. 4. Delay from the source to the destinations in MO and in SP.

where $l(x, y)$ is the longest path which can be removed by merging $T_{x}$ and $T_{y}$. Finally, a spanning tree can be constructed in $G^{\prime}$ using the Prim algorithm [13]. The resulting tree is transferred back into a forest in $G$.

\section{EXPERIMENTAL RESULTS}

The behavior of the studied algorithms were analyzed in realistic network topologies.

The Doar algorithm has been used to generate topologies [14]. Nodes are generated randomly on the Cartesian coordinate grid. Edges are added to each node pairs considering the following probability function:

$$
P_{e}(u, v)=\frac{k \bar{e}}{|G|} \beta \exp \frac{-d(u, v)}{\alpha L},
$$

where $L$ is the maximum distance between the nodes. $\alpha$ and $\beta$ parameters affect the number of connections. In the simulations, values of 0.25 and 0.2 are used for $\alpha$ and $\beta$ respectively, resembling geographical locations of the nodes in the real Internet [14]. $\bar{e}$ is the average degree of the nodes and $|G|$ is the total number of nodes in the graph. The value of the $k$ parameter is set to 25 . Values of $\alpha, \beta$ and $k$ are choosen according to the Doar article [14]. Unconnected topologies are sorted out.

The algorithms are implemented in $\mathrm{C}++$ using LEDA functions [15]. There are 50 nodes generated at each network topology. $30 \%$ of the nodes were multicast members, they were choosen randomly. Each measurement was repeated for 1000 times for 11 different splitting capability percentages.

From the Figure 4, it can be seen that the average source to member delay is constant for the SP heuristic. There is no relation between the percentage of splitting capable nodes and the delay. To keep this property, the heuristic has to construct more trees. The delay of MO heuristic greatly depends of the percentage of splitting incapable nodes. In constrained networks, this heuristic 


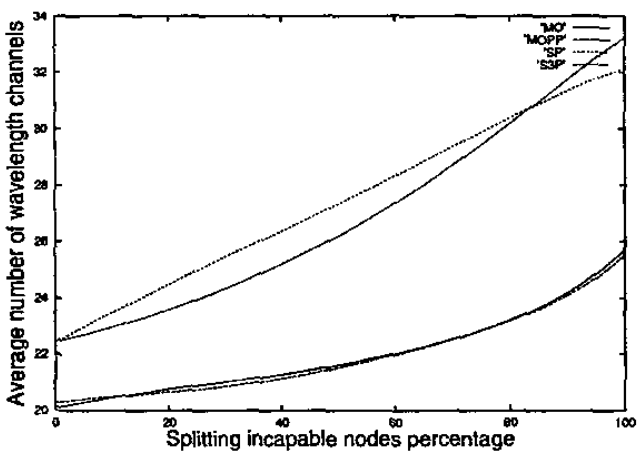

Fig. 5. Number of wavelength channels in MO, SP, S3P and MOPP

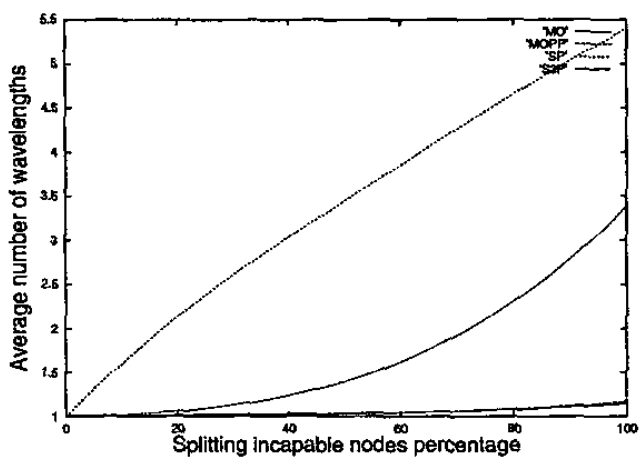

Fig. 6. Number of wavelength in MO, SP, S3P and MOPP

extends the tree on the leaf nodes, increasing the average delay from the source to the destinations. The delay of MO is always significantly bigger than the delay of SP, but in the optical domain, the delay is not a serious limitation.

The number of wavelength channels used for both heuristics increases with the percentage of splitting incapable nodes (see Figure 5). The average number of wavelength channels for SP is up to 7 more than for MO. Edges close to the source have higher probability to be used by more trees than other edges. Figure 6 shows this behavior. This behavior has to be improved to be useful in optical networks.

Simulations were done on both SP and S3P and the number of wavelengths and wavelength channels were compared. Results are shown on figure 5 and 6 . Using PP algorithm the number of wavelength channels is reduced between $0 \%$ and $80 \%$ splitting incapability. The number of wavelengths is also greatly reduced using PP algorithm, with a maximum reduction of a factor of 3. Using PP also reduced greatly the number of wavelengths maximum by three times.
The PP algorithm was also applied for the MO heuristic (MOPP). The obtained results are shown in Figure 5 and 6. There is no significant difference between them in terms of the number of wavelength channels. Below $80 \%$, the number of wavelengths used by $\mathrm{MO}$ is reduced but only with $1 \%$.

\section{Conclusions}

Shortest Path Based Forest heuristics were introduced for sparse splitting capable optical networks. A Post Processing algorithm was proposed to reduce the number of wavelengths and wavelength channels on a specific heuristic. It has also been tested on a well-known heuristic, called Member-Only. Results show that the PP algorithm reduces the number of wavelengths used. With the proposed modification, the S3P algorithm becomes a good candidate for multicast routing protocols in alloptical networks.

\section{REFERENCES}

[1] K. Fukuchi, T. Kasamatsu, M. Morie, and R. Ohhira. 10.92-TB/s $(273 \times 40-\mathrm{GB} / \mathrm{s})$ triple-band/ultradense WDM optical-repeatered transmission experiment. Technical Digest Optical Fiber Communication Conference, 2001.

[2] J. Moy. Multicast extensions to OSPF. Internet Requests for Comments (RFC) 1075, 1991.

[3] N. K. Singhal and B. Mukherjee. Architectures and algorithm for multicasting in WDM optical mesh networks using opaque and transparent optical cross-connects. Proc., OFC 2001, Anaheim $C A, 2001$.

[4] M. Ali and J. S. Deogun. Cost-effective implementation of multicasting in wavelength-routed networks. Journal of Lighwave Technology, VOL, 18, NO. 12., December, 2000.

[5] X. Zhang R. Malli and C. Qiao. Benefit of multicasting in alloptical networks. Procedings of SPIE, All Optical Networking, pages 202-209, 1998.

[6] L. H. Sahasrabuddhe and B. Mukheriee. Light-trees: Optical multicasting for improved performances in wavelength routed networks. IEEE Communications Magazine, 37(2):1950-1960, 2000.

[7] C.S.R. Murthy and M. Gurusamy. WDM optical networks concepts, design, and algorithms. Prentice-Hall, Inc., ISBN O13-060637-5, 2002.

[8] S. Voss. Problems with generalized steiner problems. Algorithmica, 7(2,3):333-335, 1992.

[9] S. Voss. Steiner-probleme in graphen. Frankfur/Main: Hain, pages 179-184, 1990.

[10] E. Dijkstra. A note on two problems in connection with graphs. Numerische Mathematik, 1:269-271, 1959.

[11] R.M. Karp. Redacibility among Combinatorial Problems. Com plexity of Computer Computations, pages 85-103, 1972.

[12] H. Takahashi and A. Matsuyama. An approximate solution for the steiner problem in graphs. Mathematica Japonica, 24, 1981,

[13] R.C. Prim. Shortest connection networks and some generalizations. The Bell System Technical Journal, 36:1389-1401, 1957

[14] M. Doar and 1. Lestie. How bad is naive multicast routing ? INFOCOM, pages 82-89, 1993.

[15] LEDA. Library of efficient data types and algorithms, http:/www.mpi-sb.mpg.de/leda/leda.html, version 3.6.1. 